\title{
Improving Non-Destructive Test Results Using Artificial Neural Networks
}

\author{
Yi-Fan Shih, Yu-Ren Wang, Shih-Shian Wei, and Chin-Wen Chen
}

\begin{abstract}
In the construction industry, non-destructive testing (NDT) methods are gaining more popularity for their ability to examine the in-situ component properties without damaging the structure. One of the most common NDTs for measuring the concrete compressive strength on site is the Rebound Hammer Test. Using the rebound value obtained from the test hammer, the concrete compressive strength can be estimated using the conversion chart provided by the instrument manufacturer. Despite for its convenience, rebound hammer test estimations have an average of over $20 \%$ mean absolute percentage error when comparing to the compressive strength obtained by destructive tests. In light of this, this research proposes an alternative approach to obtain the concrete compressive strength using the rebound value from the test hammer. That is, by applying the Artificial Neural Networks (ANNs) to develop a prediction model for concrete compressive strength estimation. Data collected from 838 lab Rebound Hammer tests are collected to train and validate the ANNs model. The ANNs model prediction results have successfully reduced the average mean absolute percentage error to $7.27 \%$. It is recommended that Artificial Neural Networks can be applied to improve non-destructive test (rebound hammer test) results.
\end{abstract}

Index Terms - Non-destructive test, compressive strength, rebound hammer test, SilverSchmidt electronic test hammer, artificial intelligence, artificial neural networks.

\section{INTRODUCTION}

In the construction industry, non-destructive testing (NDT) methods are gaining more popularity for their ability to examine the in-situ component properties. NDTs are typically much cheaper and faster comparing to traditional destructive tests. In addition, the NDT will not cause any damage to the structure components, especially true when one wants to estimate the onsite concrete strength. One of the most common NDTs for measuring the concrete compressive strength on site is the Rebound Hammer Test [1]. Using the rebound value obtained from the test hammer, the concrete compressive strength can be estimated almost instantly using the conversion chart provided by the instrument manufacturer [2]. Despite for its convenience, rebound hammer test results have shown that the estimation has an average of over $20 \%$ mean absolute

Manuscript received on May 9, 2015; revised August 3, 2015.

Yi-Fan Shih and Chin-Wen Chen are with the Civil Engineering \& Disaster Prevention Technology Institute, National Kaohsiung University of Applied Sciences, Chien-Kung Road, Kaohsiung, Taiwan (e-mail: shih090202@kimo.com, jessica199111@gmail.com)

Yu-Ren Wang was with the University of Texas at Austin. He is now with the National Kaohsiung University of Applied Sciences, Chien-Kung Road, Kaohsiung, Taiwan (e-mail: yrwang@ kuas.edu.tw).

Shih-Shian Wei was with the Civil Engineering \& Disaster Prevention Technology Institute, National Kaohsiung University of Applied Sciences, Chien-Kung Road, Kaohsiung, Taiwan (e-mail: 1sk1@ms15.hinet.net). percentage error when comparing to the compressive strength obtained by destructive tests [3]. Due to this major drawback, rebound hammer test results are often treated as reference only, unless under the circumstance that obtaining destructive test results is structurally non-feasible. In light of this, this research proposes an alternative approach to obtain the concrete compressive strength using the rebound value from the test hammer. That is, by applying the Artificial Intelligence (AI) technique to improve the concrete strength estimation instead using the conversions provided by the instrument manufacturer. It has been shown in many studies that Artificial Intelligence techniques demonstrate better ability in data regression and prediction when comparing to the traditional statistical methods such as linear and non-linear regression [4]. Among them, Artificial Neural Networks (ANNs) is a popular machine learning techniques that has been successfully applied in many areas for classification and regression [5]. Collaborating with a professional material testing laboratory, information are collected from 838 lab Rebound Hammer tests. The collected data are used to train and validate the ANNs model for concrete compressive strength prediction. The ANNs model prediction results have shown significant improvement and successfully reduce the average mean absolute percentage error to $7.27 \%$. It is recommended that Artificial Neural Networks can be applied to improve non-destructive test (rebound hammer test) results.

\section{RELATED RESEARCH}

In order to explore the possibility of proposing an Artificial Neural Networks (ANNs) model to improve the non-destructive tests, related literature concerning the Rebound Hammer Test and ANNs are reviewed and summarized below.

Rebound Hammer Test. As one of the most popular non-destructive tests, Rebound Hammer test provides a convenient and rapid way to measure the concrete compressive strength. The basic principle of the rebound hammer test is that the rebound of an elastic mass depends on the hardness of the surface the mass strikes. The extent of the rebound is an indication of the surface hardness of the test object. In the case of the concrete test, low strength and stiffness concrete will yield a lower rebound value due to more energy absorption [6]. During the rebound hammer test, the plunger of the rebound hammer is first pressed against the surface of the concrete, and then a gradual increase in pressure is applied until the hammer impacts. The rebound value is read from a graduated scale and is designated as the rebound number or rebound index (Q- 
value). The concrete compressive strength can be estimated using the conversion table provided by the manufacturer [7]. Fig. 1 illustrates this process.

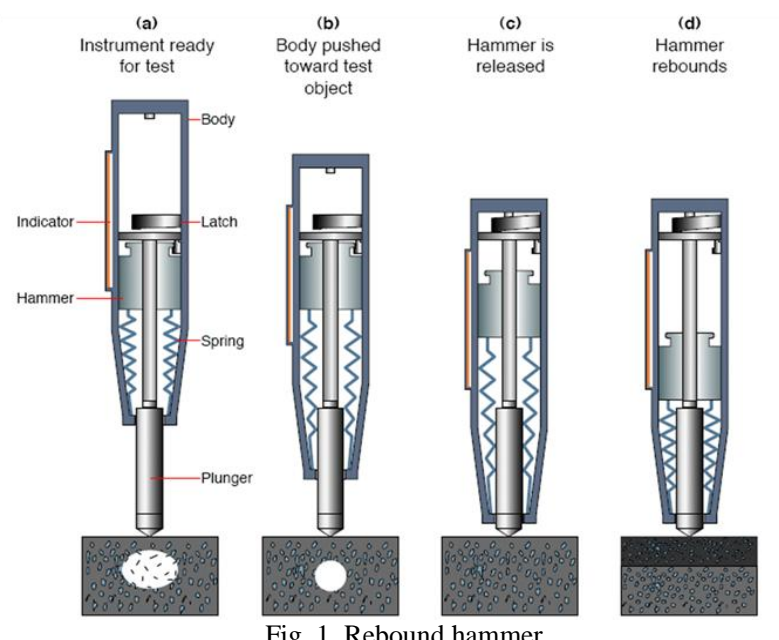

For this research, a material testing lab is chosen for the rebound hammer test data collection. Ten rebound hammer tests are conducted evenly distributed on top of the circular concrete cylinder samples $(15 \mathrm{~cm} \times 30 \mathrm{~cm})$. A total of 838 samples are collected during a four-month period. The rebound values are recorded and their corresponding compressive strength estimations are also obtained. After the rebound hammer test, destructive tests are conducted on these test samples to acquire the actual concrete compressive strengths. The data collected are used to develop, validate and test the Artificial Neural Networks model.

Artificial Neural Networks. Comparing to traditional statistical methods such as multiple regression analysis and multivariate analysis, Artificial Neural Networks (ANNs) are proven to be more effective in dealing with non-linear data. The sample data are not required to follow a specific statistical distribution; neither should the relationships between inputs and outputs be predetermined. ANNs are very capable of learning and self-updating through the training process [8]. As a result, this research adopts ANNs to develop an AI model to improve the Non-Destructive Test prediction.

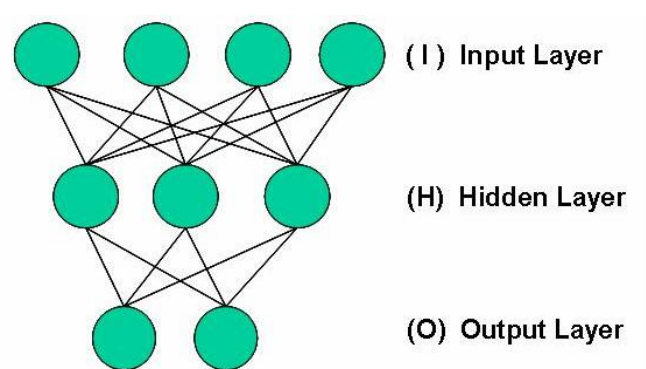

Fig. 1. Typical three-layer neural networks.

The development of ANNs is inspired by the animal central nervous systems (biological neural networks). The principle of Neural Networks is based on the assumption that a highly interconnected system of simple processing elements can learn complex interrelationships between independent and dependent variables, just like the interconnected neurons in the brain [9]. A typical neural network consists of an input layer, an output layer, and one or more hidden layers. These layers are connected by neurons to form a parallel distributed processing system. Each neuron is viewed as a processing element (PE) that receives inputs and generates outputs through an activation function. Each of the connections between the process elements has an associated weight. Fig. 2 shows a typical three-layered neural network with an input layer (I), a hidden layer $(\mathrm{H})$, and an output layer $(\mathrm{O})$.

In the hidden layer, each neuron receives an activation signal (input), and generates a signal (output) through an activation function. The activation signal is the weighted sum of all the signals entering the neuron, as shown in Equation 1. In Equation 1, $x_{j}$ is the activation signal that the neuron $j$ in the hidden layer receives; $I_{i}$ is $i$ th input in the input layer; and $W_{i j}$ is the weight of the connection between the neuron $j$ in the hidden layer and the input $I_{i}$. The neuron (Process Element) produces an output through an activation function that can be any form. The activation function can be either linear or non-linear, and one of the most commonly used activation function is the sigmoid function. The general form of sigmoid function is shown in Equation 2 , where $h_{j}$ equals output of the neuron $j$ in the hidden layer and $x_{j}$ equals input for the neuron $j$ [9].

$$
\begin{gathered}
x_{j}=\sum_{i} I_{i} W_{i j} \\
h_{j}=f\left(x_{j}\right)=\frac{1}{1+e^{-x_{j}}} \\
y_{k}=\sum_{j} h_{j} W_{j k}
\end{gathered}
$$

As presented by Equation 3, the neurons in the output layer receive activation signals (weighted sum of inputs to neuron $\mathrm{k}$ ) from the neurons in the hidden layer. In Equation $3, y_{k}$ is the input of the neuron $k$ in the output layer and $W_{j k}$ is the weight of the connection between the neurons $j$ and $k$ in the hidden and output layers, respectively. In the output layer, these activation signals are transformed (through activation function) again to generate the outputs of the neural network. This process is shown in Equation 4, where ok is the predicted value of the outputs. Then the outputs are compared with desired or actual values, $d_{k}$. The error (difference between predicted value and desired/actual value) at the output neurons is defined by Equation 5 . The best performance of the neural network is achieved when the error is minimized [9].

$$
\begin{aligned}
& o_{k}=f\left(y_{k}\right)=\frac{1}{1+e^{-y_{k}}} \\
& E(W)=\frac{1}{2} \sum_{k}\left(d_{k}-o_{k}\right)^{2}
\end{aligned}
$$

For supervised neural networks (models with specific actual/desired outputs), one of the most effective and popular technique to minimize the error function $E(W)$ is the back-propagation (BP) algorithm. For back-propagation neural networks, the error at the output layer propagates backward to the hidden layer and then to the input layer to update the weights for each of the connections in the neural networks. These forward process (input layer to hidden 
layer to output layer) and backward process (output layer to hidden layer to input layer) are repeated to minimize the error [9].

These repeated processes are viewed as learning (training) process. The relationships between inputs and outputs of the system are memorized through the connection weights. It should be noted that before the learning process starts, small random numbers (e.g., between -0.1 and 0.1 ) are assigned as the initial weights to the connections between the neurons. This ensures that the network is not saturated by large values of the weight, and prevents some training pathologies. Sometimes, the data will be normalized before to obtain convergence within a reasonable number of cycles [9].

For this research, the ANNs model is developed using NeuroSolution ${ }^{\circledR}$ software.

\section{DATA COLleCtion AND MODEl DEVElopment}

The researchers collaborate with a local professional material testing lab, which is a government certified laboratory conducting various destructive and nondestructive test on construction materials. For the purpose of this research, ten Rebound Hammer Test readings are taken for each concrete cylinder test sample $(15 \mathrm{~cm}$ in diameter and $30 \mathrm{~cm}$ in height) using SilverSchmidt N-Type electronic rebound hammer manufactured by Proceq. These ten measurements are taken evenly distributed on top of the concrete cylinders and then recorded in the database. After the Rebound Hammer Tests, the samples went through destructive compressive strength tests using HT-8391 200 ton concrete compression test machine. Data from a total of 838 samples are collected and used for ANNs prediction model development and validation.

TABLE I: ANNS MODEL PARAMETER

\begin{tabular}{|c|c|c|}
\hline Model Parameter & \multicolumn{2}{|c|}{ Contents } \\
\hline ANNs Model & \multicolumn{2}{|c|}{ Backpropagation Network } \\
\hline \multirow{2}{*}{ Exemplars } & Training Dataset & 670 \\
\hline & Testing Dataset & 168 \\
\hline $\begin{array}{l}\text { No. Of Hidden } \\
\text { Layer }\end{array}$ & \multicolumn{2}{|c|}{1 and 2} \\
\hline $\begin{array}{c}\text { No. of Processing } \\
\text { Elements }\end{array}$ & \multicolumn{2}{|c|}{ Constructive Algorithm } \\
\hline Transfer Function & \multicolumn{2}{|c|}{ TanhAxon } \\
\hline Learning Rule & \multicolumn{2}{|c|}{ Levenberg Marqua } \\
\hline Maximum Epochs & \multicolumn{2}{|c|}{ Default: 5000} \\
\hline Termination & \multicolumn{2}{|c|}{$\begin{array}{l}\text { 1. Minimum MSE } \\
\text { 2. Maximum Epochs }\end{array}$} \\
\hline
\end{tabular}

ANNs are developed in the NeuroSolutions ${ }^{\circledR} 6.0$ software. Among the 838 sample data, 670 of them are randomly chosen and set as the training dataset to train the ANNs model. The remaining 168 of them are set as testing dataset to validate the model developed. For the ANNs model development, the researchers explored various parameter combinations to obtain the best results. The model parameters include number of hidden layer, number of processing elements in the hidden layer, type of transfer function and learning rule, and number of training epochs.
Table I summarizes the parameter setup for the ANNs model.

To obtain better model prediction accuracy, several models are developed with different input variables and model parameter setups. At first, all ten measurements (Qvalues recorded from the electronic hammer) are taken as the model inputs (10 input variables) for the ANNs model and the actual compressive strength obtained from the destructive tests are set as the output variable. Then simpler model with only two input variables (average and standard deviation of the 10 measurements) is developed. In the meantime, the ANNs model with one and two hidden layers are also experimented with different process elements in the hidden layer. Different model types and model parameters are developed to find out the best prediction model setup.

After the model is trained with the training data, the remaining 168 samples in the dataset are used to test the trained model to obtain the compressive strength prediction accuracy. The model prediction accuracy is measured by the mean absolute percentage error (MAPE), as shown in Equation 6. The results have shown that ANNs model with two input variables yield better prediction results when comparing to models with 10 input variables. Also, models with one hidden layer outperform models with two hidden layers. Model prediction results with two input variables are summarized in the Table II below.

$$
\text { MAPE }=\left\langle\frac{1}{N} \sum_{i=1}^{N} \frac{\left|A_{i}-O_{i}\right|}{A_{i}}\right\rangle \times 100 \%
$$

where $A$ is actual compressive strength, $O$ is model output and $N$ is the number of sample data.

TABLE II: ANNS MODEL PREDICTION RESULT

\begin{tabular}{|c|c|c|c|}
\hline Network Type & No. of Hidden Layer & Epochs & MAPE \\
\hline $2-2-1$ & 1 & 5000 & $7.47 \%$ \\
\hline $2-2-2-1$ & 2 & 5000 & $8.20 \%$ \\
\hline $2-2-1$ & 1 & 10000 & $7.27 \%$ \\
\hline $2-2-2-1$ & 2 & 10000 & $7.68 \%$ \\
\hline
\end{tabular}

As shown in Table II, the best MAPE obtained is $7.27 \%$ for the 2-2-1 back propagation artificial neural networks (2 inputs, one hidden layer with two process elements, and one output). In the meantime, the MAPE of the 168 test samples for the estimation from SilverSchmidt N-Type electronic rebound hammer is $24.77 \%$. It is evident that the proposed ANNs model is able to produce much better predictions of the concrete compressive strength comparing to the SilverSchmidt hammer test estimations.

\section{CONCLUSIONS AND RECOMMENDATIONS}

For this research, an Artificial Intelligence technique (Artificial Neural Networks) is proposed to improve the concrete compressive strength estimation for the Rebound Hammer Test (a non-destructive test method). A total of 838 concrete cylinder samples are tested using both Rebound Hammer Test and destructive concrete compressive strength tests. The actual concrete compressive strengths are obtained through the destructive test in order 
to compare with the estimations from the Rebound Hammer Test. From the 838 test samples, 670 of them are randomly chosen as the training dataset and the remaining 168 are set as testing dataset. Ten rebound hammer test measurements are taken for each test sample and the Q-values obtained are set as the input variables for the ANNs model. Meanwhile, the concrete compressive strengths are set as the desired output. Mean absolute percentage error (MAPE) is used to examine the model prediction accuracy. Various ANNs model types and model parameters are explored to get the best prediction results. The best MAPE obtained is $7.27 \%$ from a 2-2-1 back propagation artificial neural networks model ( 2 inputs, one hidden layer with two process elements, and one output). The results show significant improvement when comparing to the MAPE obtained from the test hammer estimations, which is $24.77 \%$ for the 168 test samples. The research results show that artificial intelligence technique (Artificial Neural Networks) can be applied to improve the non-destructive test estimation, in this case, the Rebound Hammer Tests. It is recommended that other artificial intelligence techniques can be explored to improve the non-destructive test results.

\section{REFERENCES}

[1] H. Y. Qasrawi, "Concrete strength by combined nondestructive methods simply and reliably predicted," Cement and Concrete Research," vol. 30, no. 5, pp. 739-746, 2000.

[2] I. A. Basheer and M. Hajmeer, "Artificial neural networks: fundamentals, computing, design, and application," Journal of Microbiological Methods, vol. 43, no. 1, pp. 3-31, 2000.

[3] Civil Engineer. [Online]. Available: http://civilengineerspk.com/rebound-hammer-test/

[4] R. J. Schalkoff, Artificial Neural Networks, McGraw-Hill, New York, 1997.

[5] S. O. Haykin, Neural Networks and Learning Machines, Prentice Hall, 2008.
[6] S. T. Russell, J. Stuart, and P. Norvig, Artificial Intelligence: A Modern Approach, 2nd ed. Prentice Hall, New Jersey, 2003.

[7] V. M. Malhotra and N. J. Carino, Handbook on Nondestructive Testing of concrete, CRC press, 2004.

[8] W. L. Huang, C. Y. Chang, W. C. Chen, and C. N. We, "Using ANNs to improve prediction accuracy for rebound hammers," Taiwan Highway Engineering, vol. 37, no. 2, pp. 2-18, 2001.

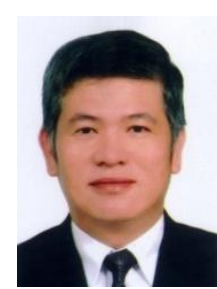

Yi-Fan Shih was born in Taiwan in 1962. He received the B.E. degree in civil and construction engineering from National Taiwan University of Science and Technology in Taiwan in 1986, and the M.S. degree in civil engineering from National Central University in Taiwan in 2001 .

$\mathrm{He}$ is the director of Taiwan Professional Civil Engineers Association, Chinese Union of Professional Civil Engineers Association and Rui Cheng Technical

Consultant Co., Ltd.

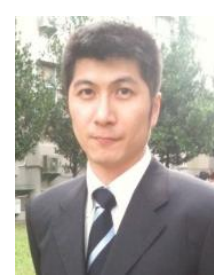

Yu-Ren Wang was born in Taiwan in 1974. He received the B.E. degree in civil engineering from National Chiao Tung University, Taiwan, in 1996, and the M.S. degree in civil and environmental engineering from University of Illinois, UrbanaChampaign, Illinois, in 1998, and the Ph.D. degree in civil engineering from University of Texas at Austin, Austin, Texas in 2002.

Since 2007, he has been with the Department of Civil Engineering in National Kaohsiung University of Applied Sciences, Kaohsiung, Taiwan, where he was an assistant professor and then became an associate professor in 2010 .

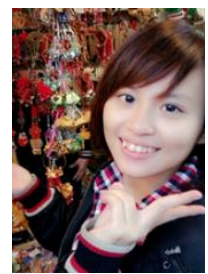

Chin-Wen Chen was born in Taiwan in 1991. She received the B.E. degree in civil and ecological engineering from I-Shou University, Taiwan in 2013. 